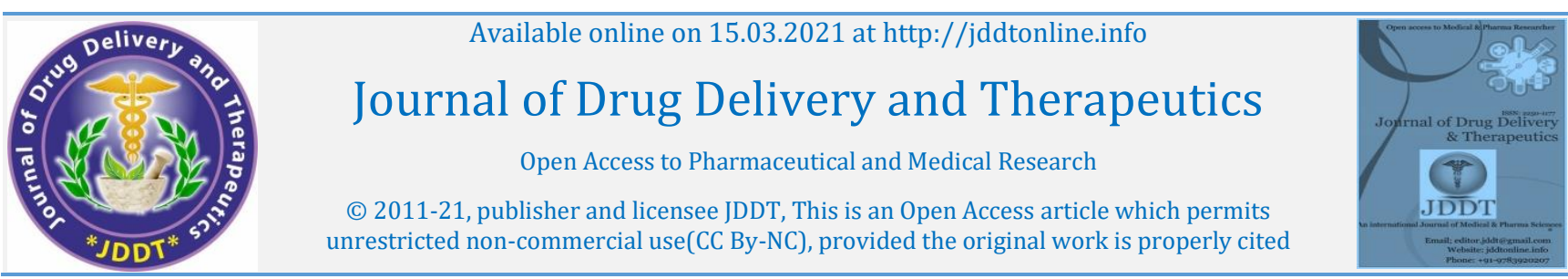

(C) 2011-21, publisher and licensee JDDT, This is an Open Access article which permits (2) Access Full Text Article

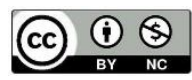

Review Article

\title{
Highlights on the alternatives to antibiotic therapy against bacterial infection
}

\author{
Bijayanta Sircar, Shyamapada Mandal* \\ Laboratory of Microbiology and Experimental Medicine, Department of Zoology, University of Gour Banga, Malda-732103, India
}

\section{Article Info:}

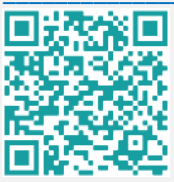

Article History:

Received 11 Feb 2021;

Review Completed $21 \mathrm{Feb} 2021$

Accepted 04 March 2021;

Available online 15 March 2021

Cite this article as:

Sircar B, Mandal S, Highlights on the alternatives to antibiotic therapy against bacterial infection, Journal of Drug Delivery and Therapeutics. 2021; 11(2):194-203 DOI: http://dx.doi.org/10.22270/jddt.v11i2.4596

\section{Abstract}

The antibiotic resistance among gram-positive and gram-negative pathogenic bacteria is of global health concern. This has prompted the development of new effective drugs. But the discovery and development of new drugs is slow, and the emergence of resistance to such new drugs, on the other hand, is rapid as well as continuous among the bacteria. Therefore, in tackling the emergence of antibiotic resistant pathogenic bacteria finding alternative ways is vital. This communication, based on the published scientific data, summarizes the antibacterial capacity of some naturally derived agents such as honey, phytocomponents, probiotics, and antimicrobial peptides that might bring new essence in biomedicine.

Keywords: Bacterial resistance, alternative therapeutics, honey, phytomedicine, probiotics, antimicrobial peptides.

*Address for Correspondence:

Dr. Shyamapada Mandal, Professor, Department of Zoology, University of Gour Banga, Malda-732103, India E-mail: samtropmed@gmail.com

\section{Introduction}

Emergence of bacterial antibiotic resistance developed through an array of mechanisms is a severe threat to humans, and such phenomenon has been marked as an global alarming problem, which in developing countries including India, as recognised by the WHO, is reaching critical levels ${ }^{1}$. The multidrug resistant (MDR) ESKAPE (gram-positive: Enterococcus faecium and Staphylococcus aureus, and gram-negative: Klebsiella pneumoniae, Acinetobacter baumannii, Pseudomonas aeruginosa, and Enterobacter spp.) bacteria are among the most notorious to cause life threatening nosocomial infections ${ }^{2}$. The continuous antibiotic therapy as well as the lack of effective antibiotics in the existing global treatment regimen has directed to a major upsurge in antibiotic resistance ${ }^{3}$. The increasing trend of development of antibiotic resistance among pathogenic bacteria has been associated with a marked economic cost worldwide. As the consequences there are great mortality and morbidity, high treatment costs, diagnostic doubts, and deficiency of trusted conventional medicine ${ }^{2}$. Of the six notorious ESKAPE pathogens, the four gram-negative bacteria, have been associated with four main types of multidrug resistance, specifically the extended-spectrum $\beta$ lactamase-producing $K$. pneumoniae and Enterobacter spp., carbapenemase-producing $A$. baumannii and metallo- $\beta$ lactamase producing Ps. aeruginosa limiting the therapeutic choices $^{4}$. K. pneumoniae is presently developing as a noticeable opportunistic pathogen and the most challenging agent of nosocomial infections ${ }^{5}$.

Exposure of the pathogenic bacteria to antibiotics surges the risk of the emergence of carbapenem resistant
Enterobactericeae, too. Carbapenems and cephalosoprins are cause of resistance that increased the risk up to 15-fold and 6 - 29 folds, respectively ${ }^{1}$. The widespread antibiotic usage in communities and hospitals cause severe multidrug resistance among gram-negative bacteria. The ESBLmediated MDR gram-negative ESKAPE pathogens are progressively associated with several conditions that are difficult to treat in both developed and developing nations ${ }^{4}$. Current researches have shown pronounced interest in the use of alternative agents including honey, phytomedicine, probiotics, and antimicrobial peptides, in targeting the bacterial resistance corroborating their potential in the treatment of diseases caused by a large number of bacteria displaying resistance to almost all the antibiotics. This study thus provides a highlight on the antibacterial capacity of some naturally available agents, based on the scientific information published in the field.

\section{Antibacterial activity}

The indiscriminate use of antibiotics causes the development of antibiotic resistance among pathogenic bacteria leading to high morbidity and mortality from infections caused by such pathogens ${ }^{6}$. In the current times, there has been an increasing interest in exploring and evolving new antimicrobial biotherapeutics from various sources to fight bacterial resistances ${ }^{7}$. Along with the growing incidence of antibacterial resistance, complete and effective investigation is needed to look for the natural antibacterial sources, such as honey, plants, probiotics providing several active compounds having antibacterial activity that could inhibit life threatening bacterial diseases (Figure 1). 


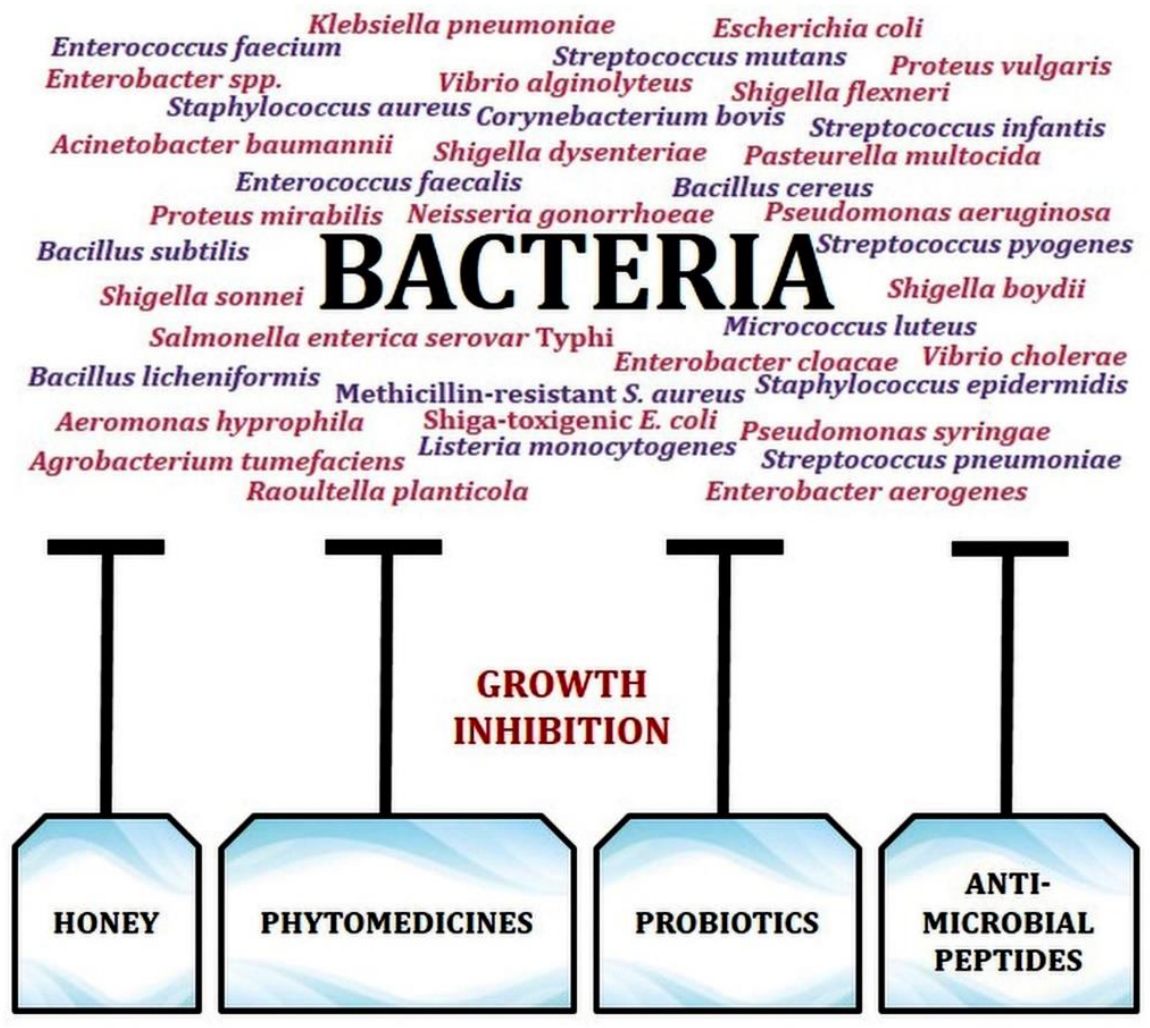

Figure 1: Schematic representation of different alternative antibacterials against human pathogenic bacteria.

\subsection{Honey}

Recently it has been proved experimentally that honey display antibacterial, anti-inflammatory and antioxidant activities, which may be useful in opposing MDR bacteria as well as in inhibiting many prolonged inflammatory processes $^{8}$. The antibacterial activity of honey against clinical isolates of Escherichia coli, Pseudomonas aeruginosa and Salmonella enterica serovar Typhi has been reported previously ${ }^{9}$. Some factors that present in the honey as antimicrobials include hydrogen peroxide $\left(\mathrm{H}_{2} \mathrm{O}_{2}\right)$ and inhibin, and also the osmotic effect of honey, its low $\mathrm{pH}$ (3.2 - 4.5), defensin-1, as well as the presence of phytochemical components display antibacterial activity ${ }^{10}$.

Most of the researchers performed the disc diffusion or well diffusion method to study the antibacterial activity of honey. Several articles on antibacterial activity of different honey samples from diverse region of the world that has been published are summarised in Table 1.

Table 1: Antibacterial activity of honey

\begin{tabular}{|c|c|c|c|c|c|c|}
\hline \multirow[t]{2}{*}{ Honey type } & \multirow[t]{2}{*}{$\begin{array}{c}\text { Geographical } \\
\text { location }\end{array}$} & \multirow[t]{2}{*}{$\begin{array}{c}\text { Using } \\
\text { condition }\end{array}$} & \multirow[t]{2}{*}{$\begin{array}{c}\text { Activity against } \\
\text { bacteria }\end{array}$} & \multicolumn{2}{|c|}{$\begin{array}{c}\text { Antibacterial } \\
\text { activity }\end{array}$} & \multirow[t]{2}{*}{$\operatorname{Ref}$} \\
\hline & & & & $\begin{array}{c}\text { ZDI } \\
(\mathrm{mm})\end{array}$ & MIC (\%) & \\
\hline $\begin{array}{c}\text { Commercial grade } \\
\text { honey }\end{array}$ & Malda, India & $\begin{array}{l}\text { Aqueous } \\
\text { honey }\end{array}$ & $\begin{array}{c}\text { Gram negative: Escherichia coli, Pseudomonas } \\
\text { aeruginosa, Proteus vulgaris, and E. coli ATCC } \\
25922 \\
\text { Gram positive: Staphylococcus aureus }\end{array}$ & $6-30$ & ND & 8 \\
\hline $\begin{array}{c}\text { Natural jujube } \\
\text { honey }\end{array}$ & Saudi Arabia & $\begin{array}{c}\text { Methanol } \\
\text { extract }\end{array}$ & $\begin{array}{c}\text { Gram negative: E. coli ATCC } 35218, \text { Klebsiella } \\
\text { pneumoniae ATCC } 700603 \text {, and K. pneumoniae } \\
\text { ATCC } 27736 \\
\text { Gram positive: } \text { S. aureus ATCC } 25923, \\
\text { Staphylococcus epidermidis ATCC } 12228, \\
\text { Enterococcus faecalis ATCC } 29212, \text { Bacillus } \\
\text { cereus ATCC } 10876,\end{array}$ & $6-17$ & ND & 11 \\
\hline $\begin{array}{l}\text { Eucalyptus honey } \\
\text { and commercial } \\
\text { grade honey }\end{array}$ & Mauritius & Undiluted & $\begin{array}{l}\text { Gram negative: Proteus sp., Klebsiella sp., } \\
\text { Pseudomonas } 161 \text { sp., and E. coli, E. coli ATCC } \\
\text { 25922, and Ps. aeruginosa ATCC } 27853 \\
\text { Gram positive: Streptococcus sp., S. epidermidis } \\
\text { ATCC 35984, and S. epidermidis ATCC } 14990\end{array}$ & $6-28$ & ND & 12 \\
\hline
\end{tabular}


Table 1: (Continued)

\begin{tabular}{|c|c|c|c|c|c|c|}
\hline \multirow[t]{2}{*}{ Honey type } & \multirow{2}{*}{$\begin{array}{c}\text { Geographical } \\
\text { location }\end{array}$} & \multirow[t]{2}{*}{$\begin{array}{l}\text { Using } \\
\text { condition }\end{array}$} & \multirow{2}{*}{$\begin{array}{l}\text { Activity against } \\
\text { bacteria }\end{array}$} & \multicolumn{2}{|c|}{$\begin{array}{l}\text { Antibacterial } \\
\text { activity }\end{array}$} & \multirow[t]{2}{*}{ Ref } \\
\hline & & & & $\begin{array}{c}\text { ZDI } \\
(\mathrm{mm})\end{array}$ & $\begin{array}{l}\text { MIC } \\
(\%)\end{array}$ & \\
\hline Blossoms honey & Slovakia & $\begin{array}{l}50 \% \text { honey } \\
\text { solution }\end{array}$ & $\begin{array}{c}\text { Gram negative: } \text { Ps. aeruginosa CCM1960 } \\
\text { Gram positive: } \text { S. aureus CCM4223 }\end{array}$ & ND & $3-27$ & 13 \\
\hline $\begin{array}{l}\text { Wildflower and bitter } \\
\text { leaf honey }\end{array}$ & Nigeria & Raw honey & $\begin{array}{l}\text { Gram negative: Salmonella typhimurium } \\
\text { ATCC } 14028, \text { Sal. typhimurium clinical, } \\
\text { Shigella dysenteriae ATCC } 11836, \text { Sh. } \\
\text { dysenteriae (clinical), E. coli ATCC } 700728, \\
\text { E. coli (clinical) } \\
\text { Gram positive: } \text { B. cereus ATCC } 14579, B \text {. } \\
\text { cereus (clinical), } \text { S. aureus ATCC } 29213 \text { and } \\
\text { S. aureus (clinical) }\end{array}$ & $6-26$ & ND & 14 \\
\hline Natural honey & Ethiopia & $\begin{array}{l}\text { Aqueous } \\
\text { honey }\end{array}$ & $\begin{array}{c}\text { Gram positive: Methicillin-resistant } S . \\
\text { aureus }\end{array}$ & $6-39$ & $\begin{array}{c}9.38- \\
37.5\end{array}$ & 15 \\
\hline $\begin{array}{l}\text { Citrus honey and } \\
\text { mango honey }\end{array}$ & Malda, India & $\begin{array}{l}\text { Aqueous } \\
\text { honey }\end{array}$ & $\begin{array}{c}\text { Gram negative: Salmonella enterica } \\
\text { serovar Typhi, Ps. aeruginosa and E. coli } \\
\text { ATCC } 25922 \\
\text { Gram positive: } \text { S. aureus }\end{array}$ & $\begin{array}{c}15- \\
35\end{array}$ & ND & 16 \\
\hline Local honey & Pakistan & $\begin{array}{l}\text { Aqueous } \\
\text { honey }\end{array}$ & $\begin{array}{c}\text { Gram negative: } E \text {. coli ATCC } 25922, \text { Ps. } \\
\text { aeruginosa ATCC } 27853 \text {, S. typhi ATCC } \\
19943 \text { and K. pneumoniae ATCC } 27736 \\
\text { Gram positive: } S \text {. aureus ATCC } 6538, \text { En. } \\
\text { faecalis ATCC } 19433\end{array}$ & $\begin{array}{c}14- \\
37\end{array}$ & ND & 17 \\
\hline $\begin{array}{l}\text { Natural (Kombu and } \\
\text { Vembu) and } \\
\text { commercial grade } \\
\text { honey }\end{array}$ & Vellore, India & $\begin{array}{l}\text { Honey } \\
\text { diluted with } \\
\text { dimethyl } \\
\text { sulfoxide }\end{array}$ & $\begin{array}{c}\text { Gram negative: E. coli, S. typhi, Proteus } \\
\text { mirabilis, K. pneumoniae, Shigella flexneri } \\
\text { and Ps. aeruginosa } \\
\text { Gram positive: } S \text {. aureus, B. cereus and } \\
\text { Enterococcus casseliflavus }\end{array}$ & $6-38$ & ND & 18 \\
\hline $\begin{array}{l}\text { Acacia, abies, } \\
\text { sideritis, herbs, } \\
\text { polyfloral and } \\
\text { conifers honeys }\end{array}$ & $\begin{array}{l}\text { Mount } \\
\text { Olympus area, } \\
\text { Greece }\end{array}$ & Raw honey & $\begin{array}{c}\text { Gram negative: } \text { A. baumannii, Citrobacter } \\
\text { freundii, K. pneumoniae, and Salmonella } \\
\text { typhimurium } \\
\text { Gram positive: } \text { Streptococcus infantis }\end{array}$ & ND & $\begin{array}{c}6.25- \\
12.5\end{array}$ & 19 \\
\hline
\end{tabular}

MIC: minimum inhibitory concentration, ND: not done, ZDI: zone diameter of inhibition

\subsection{Phytomedicines}

Roots, leaves, seeds, bark or other part of medicinal plants possess therapeutic, tonic, purgative or other pharmacologic activity under in vitro as well as in vivo conditions. Several plants are used in various countries as the source of potent and powerful medicines ${ }^{20}$. Alkaloids, norsecurinines, phyllanthine, phyllochrysine, saponins, quercetin, quercetol, rutin, quercitrin, astragalin, gallocatechins, niruretin, nirurin, brevifolin, ellagic acid ellagitannins, repandusinic acids, geraniin, carboxylic acids, corilagin, cymene, lupeols, phyllanthenol, lignans, hypophyllanthin, niranthin, nirtetralin, lintetralins, methyl salicylate, niruriside, triacontanal, tricontanol etc. type of bioactive compounds are present in various plants as the source of therapeutic components 21 .

The innovation of medicinal plants in different parts of the globe is vital to the agriculture and medicine sectors, in defining the new guidelines towards spread of unconventional medicinal crops that offer improved commercial welfares 22 . Some tribal communities are mostly dependent upon the natural resources for their traditional food habits as well as for treating common illnesses such as diarrhoea, dysentery, vomiting, headache, cold, and fever ${ }^{23}$.

Indian flora deals countless possibilities for the detection of new compounds with important medicinal uses in opposing infection. The antimicrobial compounds found in plants may inhibit bacterial toxicities by alternative mechanisms than the conventional one ${ }^{24}$. Phytomedicine, prepared from different plant materials, such as Ayurvedic traditional medicine, are relatively safe, cost effective and have less or no side effects 25 .

Most of the current in vitro study on different medicinal plants with their experimental particulars, in terms of the antibacterial activity, are summarized in Table 2, where some research on bioactive fruit plants and spice herbs are also included. 
Table 2: Antibacterial activity of different plant extracts

\begin{tabular}{|c|c|c|c|c|c|c|}
\hline \multirow[t]{2}{*}{ Plants } & \multirow{2}{*}{$\begin{array}{l}\text { Plant } \\
\text { parts }\end{array}$} & \multirow{2}{*}{$\begin{array}{l}\text { Extracting } \\
\text { solvent }\end{array}$} & \multirow{2}{*}{$\begin{array}{c}\text { Activity against } \\
\text { Bacteria }\end{array}$} & \multicolumn{2}{|c|}{ Antibacterial activity } & \multirow[t]{2}{*}{ Ref } \\
\hline & & & & $\begin{array}{c}\text { ZDI } \\
(\mathrm{mm})\end{array}$ & $\begin{array}{c}\text { MIC } \\
(\mu \mathrm{g} / \mathrm{ml})\end{array}$ & \\
\hline \multicolumn{7}{|c|}{ Medicinal plants } \\
\hline $\begin{array}{l}\text { Aegle } \\
\text { marmelous } \\
\text { (Bael) }\end{array}$ & Leaves & $\begin{array}{l}\text { Hexane, } \\
\text { acetone, } \\
\text { ethanol, and } \\
\text { aqueous }\end{array}$ & $\begin{array}{l}\text { Gram negative: } \text { E. coli, Ps. aeruginosa, } \\
\text { Salmonella enterica, Shigella sonnei } \\
\text { Gram positive: Bacillus cereus, Strep. } \\
\text { faecalis, Listeria innocua, Micrococcus } \\
\text { luteus }\end{array}$ & ND & $297-551$ & 23 \\
\hline $\begin{array}{l}\text { Azardirchata } \\
\text { indica }\end{array}$ & $\begin{array}{l}\text { Leaves and } \\
\text { bark }\end{array}$ & $\begin{array}{l}\text { Ethanol, } \\
\text { chloroform } \\
\text { and } \\
\text { methanol }\end{array}$ & $\begin{array}{c}\text { Gram negative: Aeromonas hyprophila, } A \text {. } \\
\text { hyprophila ATCC 7966, Ps. aeruginosa, } \\
\text { Proteus mirabilis, Shiga-toxigenic E. coli } \\
\text { Gram positive: } S . \text { aureus, } S \text {. aureus ATCC } \\
\text { 25923, Enterococcus faecalis, Methicillin- } \\
\text { resistant } S \text {. aureus }\end{array}$ & $6-27$ & $\begin{array}{l}500- \\
12500\end{array}$ & $26-29$ \\
\hline $\begin{array}{c}\text { Withania } \\
\text { somnifera } \\
\text { (Aswagandha) }\end{array}$ & Leaves & $\begin{array}{l}\text { Ethyl } \\
\text { acetate and } \\
\text { methanol }\end{array}$ & $\begin{array}{c}\text { Gram negative: } \text { E. coli ATCC } 25922, \\
\text { Proteus mirabilis ATCC 35659, Ps. } \\
\text { aeruginosa ATCC 27853, Pseudomonas } \\
\text { syringae pv. Phaseolicola and Xanthomonas } \\
\text { campestris pv. Phaseoli } \\
\text { Gram positive: } \text { S. aureus ATCC 25923, } \\
\text { Streptococcus pneumoniae ATCC 49619, En. } \\
\text { Faecalis ATCC } 29212\end{array}$ & $7-13$ & $\begin{array}{l}6.25- \\
2500\end{array}$ & 30,31 \\
\hline $\begin{array}{l}\text { Bacopa } \\
\text { monnieri } \\
\text { (Brahmi) }\end{array}$ & $\begin{array}{l}\text { Whole } \\
\text { plant and } \\
\text { leaves }\end{array}$ & $\begin{array}{l}\text { Methanol, } \\
\text { acetone, } \\
\text { ethanol and } \\
\text { methanol }\end{array}$ & $\begin{array}{l}\text { Gram negative: } \text { E. coli K 88, Ps. aeruginosa, } \\
\text { Salmonella typhii } 62 \text {, Shigella dysenteriae 3, } \\
\text { E. coli, K. pneumoniae and K. pneumoniae } \\
\text { MTCC } 109 \\
\text { Gram positive: S. aureus ATCC } 6571 \text {, } \\
\text { Streptococcus faecalis 52, En. faecalis ATCC } \\
\text { 29212, S. aureus MTCC } 3160 \text { and B. subtilis } \\
\text { MTCC } 441\end{array}$ & $8-22$ & $\begin{array}{c}30- \\
25000\end{array}$ & 32,33 \\
\hline $\begin{array}{l}\text { Santalum } \\
\text { album (Sandal } \\
\text { wood) }\end{array}$ & Heartwood & $\begin{array}{l}\text { n-hexane, } \\
\text { water } \\
\text { chloroform, } \\
\text { acetone, } \\
\text { butanol } \\
\text { ethylacetate } \\
\text { and } \\
\text { ethanol }\end{array}$ & $\begin{array}{l}\text { Gram negative: } \text { E. coli } 25922 \text {, E. coli } 35318 \\
\text { and Shigella sonnei BB-8 } \\
\text { Gram positive: } \text { S. aureus } 25923 \text {, S. aureus } \\
\text { 38541, Streptococcs pyrogenes Tc-11-2 and } \\
\text { Neisseria gonorrhoeae } 4 \text { c- } 11\end{array}$ & $6-17$ & ND & 34 \\
\hline $\begin{array}{l}\text { Ranwolfia } \\
\text { serpentina } \\
\text { (Sarpa } \\
\text { gandha) }\end{array}$ & $\begin{array}{l}\text { Leaves, } \\
\text { Roots and } \\
\text { leaves }\end{array}$ & $\begin{array}{l}\text { Acetone, } \\
\text { methanol } \\
\text { and ethanol }\end{array}$ & $\begin{array}{c}\text { Gram negative: } E \text {. coli and } S \text {. typhi } \\
\text { Gram positive: } S \text {. aureus, B. cereus and } B . \\
\text { subtilis }\end{array}$ & $7-22$ & $\begin{array}{l}4000- \\
9000\end{array}$ & 20,35 \\
\hline $\begin{array}{l}\text { Ocimum } \\
\text { sanctum } \\
\text { (Tulsi) }\end{array}$ & Leaves & $\begin{array}{l}\text { Aqueous, } \\
\text { acetone and } \\
\text { ethanol }\end{array}$ & $\begin{array}{l}\text { Gram negative: } \text { K. pneumoniae, E. coli, Pr. } \\
\text { vulgaris, Ps. aeruginosa, S. typhi, } \\
\text { Acinetobacter baumannii and E. coli MTCC } \\
443 \\
\text { Gram positive: Streptococcus mitis, } \\
\text { Streptococcus viridans, S. aureus, B. cereus } \\
\text { and Listeria monocytogenes MTCC } 657\end{array}$ & $6-28$ & ND & 36,37 \\
\hline $\begin{array}{c}\text { Mentha } \\
\text { pipertia } \\
\text { (Pippermint) }\end{array}$ & Leaves & $\begin{array}{l}\text { Ethanol, } \\
\text { chloroform } \\
\text { and hexane }\end{array}$ & $\begin{array}{c}\text { Gram negative: } \text { E. aerogenes and } S . \\
\text { typhimirium } \\
\text { Gram positive: } S . \text { aureus, } B \text {. subtilis and } \\
\text { Propioni bacterium acnes MTCC } 1951\end{array}$ & $7-8$ & $\begin{array}{l}312- \\
1150\end{array}$ & 38,39 \\
\hline
\end{tabular}


Table 2: (Continued)

\begin{tabular}{|c|c|c|c|c|c|c|}
\hline \multirow[t]{2}{*}{ Plants } & \multirow[t]{2}{*}{ Plant parts } & \multirow[t]{2}{*}{$\begin{array}{l}\text { Extraction } \\
\text { solvent }\end{array}$} & \multirow{2}{*}{$\begin{array}{l}\text { Activity against } \\
\text { bacteria }\end{array}$} & \multicolumn{2}{|c|}{$\begin{array}{l}\text { Antibacterial } \\
\text { activity }\end{array}$} & \multirow[t]{2}{*}{ Ref } \\
\hline & & & & $\begin{array}{l}\text { ZDI } \\
(\mathrm{mm})\end{array}$ & $\begin{array}{c}\text { MIC } \\
(\mu \mathrm{g} / \mathrm{ml})\end{array}$ & \\
\hline $\begin{array}{l}\text { Phyllanthous } \\
\text { amarus (Bhumi } \\
\text { amla) }\end{array}$ & $\begin{array}{l}\text { Whole } \\
\text { plant and } \\
\text { leaves }\end{array}$ & $\begin{array}{l}\text { Aqueous, } \mathrm{n}- \\
\text { hexane, ethyl } \\
\text { acetate and } \\
\text { methanol }\end{array}$ & $\begin{array}{c}\text { Gram negative: } E \text {. coli, Ps. aeruginosa and } \\
\text { Pseudomonas spp. } \\
\text { Gram positive: Coagulase positive } S . \\
\text { aureus and } S . \text { aureus }\end{array}$ & $9-26$ & ND & 21,40 \\
\hline $\begin{array}{l}\text { Enhydra } \\
\text { fluctuans } \\
\text { (helencha) }\end{array}$ & $\begin{array}{l}\text { Whole } \\
\text { aerial parts } \\
\text { (stem and } \\
\text { leaves) }\end{array}$ & $\begin{array}{c}\text { Methanol } \\
\text { and aqueous }\end{array}$ & $\begin{array}{l}\text { Gram negative: } \text { A. baumannii, Ps. } \\
\text { aeruginosa and E. coli ATCC25922 } \\
\text { Gram positive: } \text { B. cereus, Listeria } \\
\text { monocytogenes and L. monocytogenes } \\
\text { MTCC657 }\end{array}$ & $6-24$ & $\begin{array}{l}2500- \\
10000\end{array}$ & 41 \\
\hline \multicolumn{7}{|c|}{ Fruit plants } \\
\hline $\begin{array}{l}\text { Elaeocarpus } \\
\text { floribundus } \\
\text { (Indian olive) }\end{array}$ & $\begin{array}{l}\text { Seed and } \\
\text { mesocarp- } \\
\text { epicarp of } \\
\text { mature } \\
\text { fruits }\end{array}$ & $\begin{array}{l}\text { Ethanol and } \\
\text { aqueous }\end{array}$ & $\begin{array}{c}\text { Gram negative: } E \text {. coli, Pr. vulgaris and PS. } \\
\text { aeruginosa ATCC } 27813 \\
\text { Gram positive: } B \text {. cereus, } S . \text { aureus and } L . \\
\text { monocytogenes MTCC } 657\end{array}$ & $6-22$ & ND & 42 \\
\hline $\begin{array}{c}\text { Mimusops } \\
\text { elengi (Bakul) }\end{array}$ & Seed & Ethanol & $\begin{array}{c}\text { Gram negative: } E \text {. coli, Pr. vulgaris, } K \text {. } \\
\text { pneumonia, E. coli ATCC 25922, } K . \\
\text { pneumonia MTCC } 7407 \text { and Ps. aeruginosa } \\
\text { ATCC } 27853\end{array}$ & $7-17$ & ND & 25 \\
\hline $\begin{array}{c}\text { Syzygium } \\
\text { cumini (Jamun) }\end{array}$ & Seed & Ethanol & $\begin{array}{c}\text { Gram negative: } E \text {. coli, K. pneumonia and } \\
\text { E. coli ATCC } 25922 \\
\text { Gram positive: } \text { S. aureus and S. aureus } \\
\text { ATCC } 29213\end{array}$ & $8-15$ & ND & 43 \\
\hline $\begin{array}{c}\text { Mangifera } \\
\text { indica (Mango) }\end{array}$ & Seed & Ethanol & & $10-20$ & ND & \\
\hline $\begin{array}{c}\text { Punica } \\
\text { granatum } \\
\text { (Pomegranate) }\end{array}$ & Fruit Peel & $\begin{array}{l}\text { Ethanol and } \\
\text { aqueous }\end{array}$ & $\begin{array}{l}\text { Gram negative: } E \text {. coli, Proteus spp., } K \\
\text { pneumoniae, } P \text {. aeruginosa, } A \text {. baumannii }\end{array}$ & $6-28$ & $\begin{array}{l}2500- \\
20000\end{array}$ & 44 \\
\hline \multicolumn{7}{|c|}{ Spices } \\
\hline $\begin{array}{l}\text { Piper nigrum } \\
\text { (Black pepper) }\end{array}$ & Corn & $\begin{array}{l}\text { Ethanol and } \\
\text { chloroform }\end{array}$ & $\begin{array}{c}\text { Gram negative: } \text { E. coli, Ps. aeruginosa, } \\
\text { Klebsiella } S p \text {, Proteus Sp. } \\
\text { Gram positive: } \text { Streptococcus mutans, } \\
\text { Coagulase negative } \text { Staphylococci and } S . \\
\text { aureus }\end{array}$ & $6-29$ & ND & 45,46 \\
\hline
\end{tabular}

KOH: potassium hydroxide, MIC: minimum inhibitory concentration, ND: not done, ZDI: zone diameter of inhibition

\subsection{Probiotics}

Probiotics, in the form of lactic acid bacteria (LAB), generally the lactobacilli, might be crucial in controlling the emerging antibiotic resistant pathogenic bacteria. Probiotics have the inhibition property against bacterial pathogens, including the antibiotic resistant individuals: spoilage, food-borne and pathogenic bacteria, by producing $\mathrm{H}_{2} \mathrm{O}_{2}$, lactic acid and bacteriocin ${ }^{47}$. Sheep and goat milks and their derivatives (cheese and yoghurt) are commercially available as functional foods, which are with nutritional as well as medicinal importance, and can be selected as valid candidates having microbiological and technological qualities ${ }^{48}$. Current studies revealed that some lactic acid bacteria isolated from non-milk fermented foods act as potential probiotics with huge nutritional as well as medicinal values that might be due to the production of bacteriocins ${ }^{49,50}$. In the intestine, probiotic microorganisms compete with pathogenic bacteria in terms of nutrients and cell-surface for colonization, and can create inhibition against biofilm formation and quorum sensing properties of many pathogens $51-53$.

The milk and non-milk food-based probiotics, being isolated and characterised by the scientists from around the world, are summarized, in terms of the effectiveness against bacteria, in Table 3. 
Table 3: Antibacterial activity of probiotics

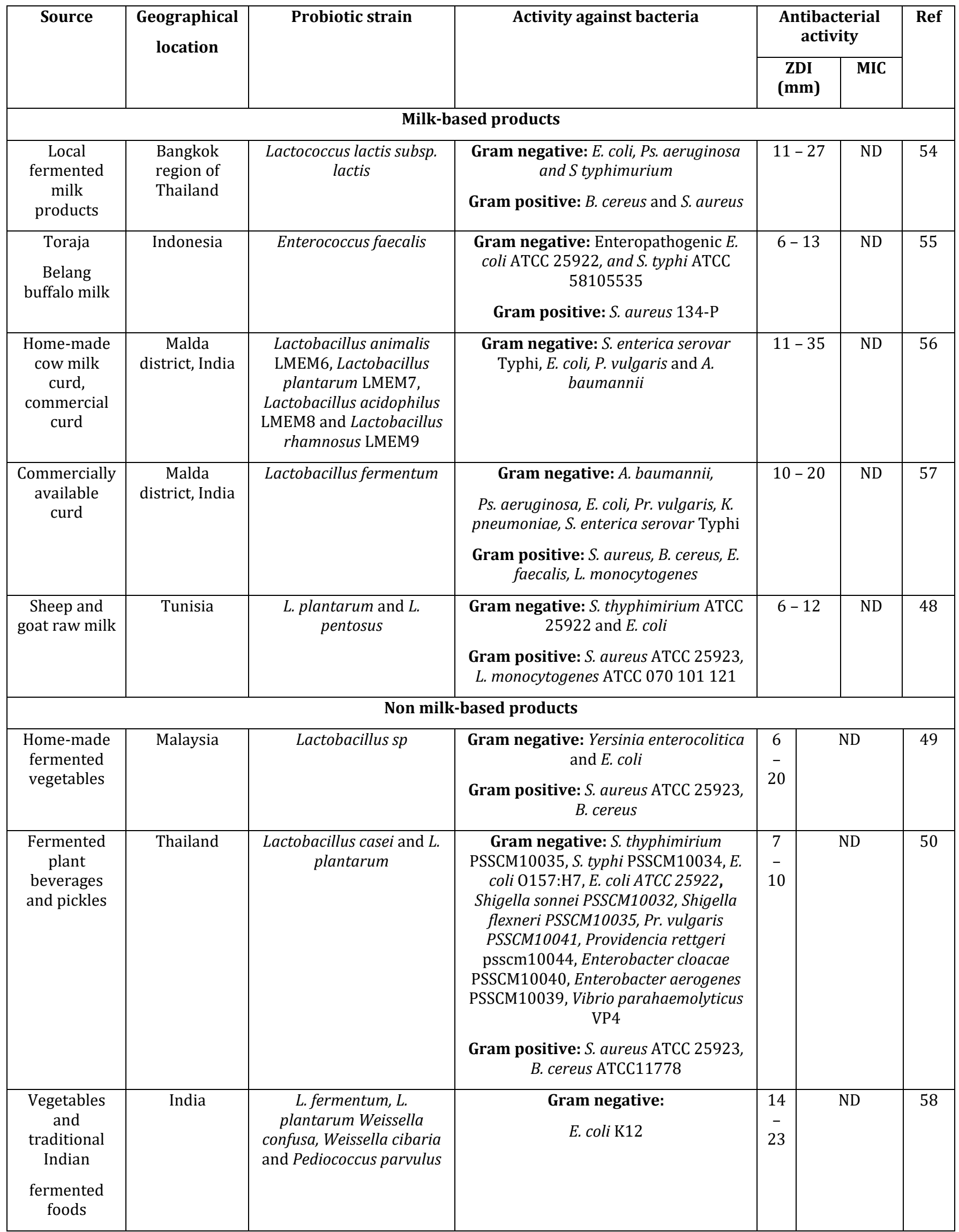

MIC: minimum inhibitory concentration, ND: not done, ZDI: zone diameter of inhibition 


\subsection{Antimicrobial peptides}

Several authors reported that antimicrobial peptides (AMPs) can be administered as typical candidates effective against different MDR bacterial strains. Biofilms formation by the bacterial cells causes more resistant to antibiotic managements than the planktonic forms of the same bacterial strains ${ }^{59}$. Food protein hydrolysates and fermented food products serves as promising source of bioactive AMPs. The caseins and whey proteins are major milk precursors proteins found in cow milk. Caseins derived bioactive peptides consists of about thirty different constituents comprising with genomic variations, mainly of $\alpha s^{-}(\alpha s 1-$, $\alpha s 2-), \beta$, and $\kappa$-casein ${ }^{60}$. Most of the potential AMPs are cationic as well as amphipathic in nature consisting of a minimum five to maximum hundred amino acids. Current studies have shown that some probiotics can synthesise AMPs that contribute significantly to host survivability, exclusively against pathogenic bacteria. Although scientists are facing some difficulties in obtaining significant and economically sustainable quantities of AMPs, and thus they are trying to manufacture heterologous endogenous AMPs using cloning technique ${ }^{61}$.
Recently, a number of anionic antimicrobial peptides have been identified in vertebrates, invertebrates and plants ${ }^{62}$. The vast source of antimicrobial peptides is marine organisms because of their close contact with microbes ${ }^{59}$. Some antimicrobial peptides derived from plants are mostly composed of cystine-rich peptides. Insects is one of the major sources of antimicrobial peptides that show inhibition against bacteria, fungi, viruses as well as some parasites. These can be classified into four families: the $\alpha$-helical peptides (cecropin and moricin), glycine-rich peptides (gloverin and attacin), proline-rich peptides (drosocin, apidaecin and lebocin) and cysteine-rich peptides (insect drosomycin and defensin) ${ }^{63}$.

Recent studies showed antimicrobial peptides can potentially serve as novel antimicrobial agents. Different AMPs can be utilized by innate immune cells and proteins to counterbalance microbial infections, and contribute more to other cellular and/or biomolecular pathways ${ }^{64}$. Table 4 summarizes the antibacterial activities of AMPs with molecular weight ranging from 1.55 to $41.44 \mathrm{kDa}$.

Table 4: Antibacterial activity of different bioactive peptides

\begin{tabular}{|c|c|c|c|c|c|c|c|}
\hline \multirow[t]{2}{*}{ Source } & \multirow{2}{*}{$\begin{array}{c}\text { Amino acid } \\
\text { number in } \\
\text { peptides }\end{array}$} & \multirow{2}{*}{$\begin{array}{c}\text { Molecular } \\
\text { weight } \\
\text { (kDa) }\end{array}$} & \multirow[t]{2}{*}{ Activity against bacteria } & \multicolumn{3}{|c|}{ Antibacterial activity } & \multirow[t]{2}{*}{ Ref } \\
\hline & & & & $\begin{array}{c}\text { ZDI } \\
(\mathrm{mm})\end{array}$ & $\begin{array}{c}\text { MIC } \\
(\mu \mathrm{g} / \mathrm{ml})\end{array}$ & $\mathrm{AU} / \mathbf{m l}$ & \\
\hline $\begin{array}{l}\text { Sea Cucumber, } \\
\text { Holothuria } \\
\text { tubulosa }\end{array}$ & $14-36$ & $1.55-4.09$ & $\begin{array}{l}\text { Gram positive: } \text { Listeria } \\
\text { monocytogenes }\end{array}$ & ND & $\begin{array}{c}1200- \\
5000\end{array}$ & ND & 59 \\
\hline $\begin{array}{l}\text { Bacteriocin from } \\
\text { Lactococcus lactis } \\
\text { MMFII (from a } \\
\text { Tunisian dairy } \\
\text { product) }\end{array}$ & $\sim 40$ & $25-41.44$ & $\begin{array}{l}\text { Gram positive: Enterococcus } \\
\text { faecalis JH22 E. faecalis V583 } \\
\text { Listeria ivanovi BUG } 496\end{array}$ & ND & $0.05-0.1$ & $20-60$ & 65 \\
\hline $\begin{array}{l}\text { Bacteriocin } \\
\text { produced by } \\
\text { Lactobacillus } \\
\text { plantarum } \\
\text { KLDS1.0391 } \\
\text { (from fermented } \\
\text { cream from China) }\end{array}$ & ND & $\begin{array}{c}21.80- \\
29.70\end{array}$ & $\begin{array}{c}\text { Gram negative: } \text { Salmonella } \\
\text { typhimurium }\end{array}$ & ND & ND & 80 & 66 \\
\hline $\begin{array}{l}\text { Marine Ascidian } \\
\text { Didemnum sp. }\end{array}$ & ND & $<40$ & $\begin{array}{l}\text { Gram negative: Ps. aeruginosa } \\
\text { ATCC 27853, Salmonella } \\
\text { typhimurium ATCC } 202165 \\
\text { Gram positive: Staphylococcus } \\
\text { aureus ATCC 6538, Serratia } \\
\text { marcescens ATCC } 14756 \text { and E. } \\
\text { faecalis ATCC } 29212\end{array}$ & $7-11$ & $\begin{array}{l}1.83- \\
2.30\end{array}$ & ND & 67 \\
\hline $\begin{array}{l}\text { Soybean, Glycine } \\
\max \end{array}$ & ND & $<10$ & $\begin{array}{c}\text { Gram negative: Acinetobacter } \\
\text { genomospecies, Aeromonas } \\
\text { hydrophila FDA110-36, A. } \\
\text { hydrophila ATCC7966, } \\
\text { Escherichia coli DH5alf, E. coli } \\
\text { ATCC43895, E. coli NCTC8959, } \\
\text { Salmonella enterica ATCC12325, } \\
\text { S. enterica ATCC29934, Vibrio } \\
\text { parahaemolyticus ATCC17802 } \\
\text { Gram positive: } \text { S. aureus } \\
\text { ATCC14458, coagulase-negative } \\
\text { S. saprophyticus KT955005, S. } \\
\text { aureus ATCC13150 }\end{array}$ & ND & $72-1050$ & ND & 68 \\
\hline
\end{tabular}


Table 4: (Continued)

\begin{tabular}{|c|c|c|c|c|c|c|c|}
\hline \multirow[t]{2}{*}{ Source } & \multirow{2}{*}{$\begin{array}{c}\text { Amino acid } \\
\text { number in } \\
\text { peptide }\end{array}$} & \multirow{2}{*}{$\begin{array}{c}\text { Molecular } \\
\text { weight } \\
\text { (kDa) }\end{array}$} & \multirow[t]{2}{*}{ Activity against bacteria } & \multicolumn{3}{|c|}{ Antibacterial activity } & \multirow[t]{2}{*}{ Ref } \\
\hline & & & & $\begin{array}{c}\text { ZDI } \\
(\mathrm{mm})\end{array}$ & $\begin{array}{c}\text { MIC } \\
(\mu \mathrm{g} / \mathrm{ml})\end{array}$ & $\mathrm{AU} / \mathrm{ml}$ & \\
\hline Laba garlic & $5-6$ & $4-6$ & $\begin{array}{c}\text { Gram negative: } \text { E. coli, ATCC } \\
\text { 25922, } \text { S. enteritidis } \\
\text { BNCC103134, } \\
\text { Gram positive: } \text { B. subtilis ATCC } \\
\text { 6633, and } S \text {. aureus ATCC } 25923\end{array}$ & $9-27$ & $\begin{array}{c}100- \\
450\end{array}$ & ND & 69 \\
\hline $\begin{array}{l}\text { Skin Secretion of } \\
\text { the Fujian Large } \\
\text { Headed Frog, } \\
\text { Limnonectes } \\
\text { fujianensi }\end{array}$ & 33 & ND & $\begin{array}{c}\text { Gram negative: } E \text {. coli NCTC } \\
10418 \\
\text { Gram positive: } S . \text { aureus NCTC } \\
10788\end{array}$ & ND & $16-32$ & ND & 70 \\
\hline $\begin{array}{l}\text { Moss } \\
\text { Physcomitrella } \\
\text { patens }\end{array}$ & $14-18$ & ND & $\begin{array}{c}\text { Gram negative: } \text { E. coli } \mathrm{K}-12 \\
\text { substr. MG1655 } \\
\text { Gram positive: } \text { B. subtilis } \\
\text { 168HT }\end{array}$ & ND & $16-128$ & ND & 71 \\
\hline $\begin{array}{l}\text { Trianthema } \\
\text { portulacastrum } \\
\text { Leaves }\end{array}$ & ND & $5.57-23.44$ & $\begin{array}{c}\text { Gram negative: } E \text {. coli } \\
\text { Gram positive: } B . \text { subtilis and } S . \\
\text { aureus }\end{array}$ & $6-14$ & ND & ND & 72 \\
\hline Rumen microbiome & $<25$ & ND & Gram negative: $A$. baumannii & ND & $64-128$ & ND & 73 \\
\hline Rana arvalis & $13-32$ & ND & $\begin{array}{c}\text { Gram negative: } \text { E. coli ATCC } \\
\text { 25922, Acinetobacter baumannii } \\
\text { ATCC } 19606 \\
\text { Gram positive: } \text { S. aureus ATCC } \\
29213 \text { and } \text { En. faecalis ATCC } \\
29212\end{array}$ & ND & $\begin{array}{c}16->64 \\
\mu \mathrm{M}\end{array}$ & ND & 74 \\
\hline
\end{tabular}

AU/ml: arbitrary unit per millilitre, MIC: Minimum inhibitory concentration, ND: not done, ZDI: zone diameter of inhibition

\section{Concluding remarks}

Due to the problem of antibiotic inactivity, exploration of alternative new antibacterial agents is needed to combat several life-threatening infections caused by MDR bacteria. Honey, plant extracts, probiotics and AMPs can inhibit the growth of infectious bacterial pathogens, as non-antibiotic antibacterials. Although, more specific experiments are required to know the effective dose dependent pharmacokinetic nature of the explored agents.

\section{References}

1. De AS, Baveja S, D'Souza D et al. Antimicrobial resistance among commonly encountered bacteria isolated in 2013 - The ESKAPE Menace, Internal Medicine, 2015; 5(193):1-6. DOI:10.4172/2165-8048.1000193

2. Rice LB, Progress and challenges in implementing the research on ESKAPE pathogens, Infection Control \& Hospital Epidemiology, 2010; 31(1):S7-S10. DOI: 10.1086/655995

3. Santajit S, Indrawattana N, Mechanisms of antimicrobial resistance in ESKAPE pathogens,

BioMed Research International, 2016; Article ID 2475067, 8 pages. DOI: $10.1155 / 2016 / 2475067$

4. Founou RC, Founou LL, Essack SY, Extended spectrum betalactamase mediated resistance in carriage and clinical gramnegative ESKAPE bacteria: a comparative study between a district and tertiary hospital in South Africa, Antimicrobial Resistance \& Infection Control, 2018; 7(134):1-11. DOI: 10.1186/s13756-018-0423-0

5. Blin C, Passet V, Touchon M et al., Metabolic diversity of the emerging pathogenic lineages of Klebsiella pneumoniae, WileyBlackwell and Society for Applied Microbiology, 2017; 19(5):1881-98. DOI: 10.1111/1462-2920.13689
6. Azzahra S, Parisa N, Fatmawati et al., Antibacterial efficacy of Aloe vera sap against Staphylococcus aureus and Escherichia coli, Bioscientia Medicina 2019; 3(2):29-37.

7. Haque SD , Saha SK, Salma U et al., Antibacterial effect of Aloe vera (Aloe barbadensis) leaf gel against Staphylococcus aureus, Pseudomonas aeruginosa, Escherichia coli and Klebsiella pneumoniae, Mymensingh Medical Journal, 2019; 28(3):490496.

8. Saha A, Mandal S, In vitro Assessment of two commercial honey samples for antibacterial and antioxidant activities, Austin Journal of Tropical Medicine \& Hygiene, 2015; 1(1):1-5.

9. Mandal S, Mandal MD, Pal NK et al., Antibacterial activity of honey against clinical isolates of Escherichia coli, Pseudomonas aeruginosa and Salmonella enteric serovar Typhi. Asian Pacific Journal of Tropical Medicine, 2010; 3(12):961-964. DOI: 10.1016/S1995-7645(11)60009-6

10. Hegazi AG, Abd Allah FM, Antimicrobial activity of different Saudi Arabia honeys, Global Veterinaria, 2012; 9(1):53-59. DOI: 10.14202/vetworld.2017.233-237

11. Abdallah EM, Hamed AE, Screening for antibacterial activity of two jujube honey samples collected from Saudi Arabia, Journal of Apitherapy, 2019; 5(1):6-9. DOI: 10.5455/ja.20190120035814

12. Aumeeruddy MZ, Aumeeruddy-Elalfi Z, Neetoo $\mathrm{H}$ et al. Pharmacological activities, chemical profile, and physicochemical properties of raw and commercial honey, Biocatalysis and Agricultural Biotechnology, 2019; 18:101005. DOI: 10.1016/j.bcab.2019.01.043.

13. Bucekova M, Jardekova L, Juricova V et al., Antibacterial activity of different blossom Honeys: New findings. Molecules, 2019; 24(1573):1-20. DOI: $10.3390 /$ molecules 24081573

14. John-Isa JF, Adebolu TT, Oyetayo VO, Antibacterial effects of Honey in Nigeria on selected Diarrhoeagenic bacteria, South Asian Journal of Research in Microbiology, 2019; 3(2):1-11. DOI: 10.9734/sajrm/2019/v3i230083 
15. Mama M, Teshome T, Detamo J, Antibacterial activity of Honey against Methicillin-Resistant Staphylococcus aureus: A laboratory-based experimental study, International Journal of Microbiology, 2019; Article ID 7686130, 9 pages, DOI: $10.1155 / 2019 / 7686130$

16. Roy S, Mandal M, Pal NK et al., Exploration of antibacterial and antioxidative property of two natural Honey samples from Malda District, India, Translational Medicine (Sunnyvale) 2016; 6(4): DOI: 10.4172/2161-1025.1000187

17. Shah T, Ali N, Shah Z et al., Antibacterial activity of Pakistani Honey, Pakistan Journal of Scientific and Industrial Research Series B: Biological Sciences, 2019; 62B(2):97-100.

18. Suganthi K, Saranraj P, Antibacterial and anticandidal activity of Natural and Commercial Honey - A comparative study, Asian Journal of Applied Research, 2018; 4(3):37-41. DOI: 10.20468/ajar.2018.02.06

19. Tsavea E, Mossialos D, Antibacterial activity of honeys produced in Mount Olympus area against nosocomial and foodborne pathogens is mainly attributed to hydrogen peroxide and proteinaceous compounds, Journal of Apicultural Research, 2019; 58(5):756-63. DOI: 10.1080/00218839.2019.1649570

20. Deshmukh SR, Ashrit DS, Patil BA, Extraction and evaluation of indole alkaloids from Rauwolfia Serpentina for their antimicrobial and antiproliferative activities, International Journal of Pharmacy and Pharmaceutical Sciences, 2012; 4(5):329-334.

21. Shrivastav A, Shrivastav N, Antibacterial activity of Bhui amla (Phyllanthus niruri), Pharma Science Monitor, 2017; 8(4):222228.

22. Zaidan MRS, Noor Rain A, Badrul AR et al., In vitro screening of five local medicinal plants for antibacterial activity using disc diffusion method, Tropical Biomedicine, 2005; 22(2):165-170.

23. Panda SK, Mohanta YK, Padhi L et al., Antimicrobial activity of select edible plants from Odisha, India against food-borne pathogens, LWT - Food Science and Technology, 2019; 9(4): 435. DOI: 10.1016/j.lwt.2019.06.013

24. Yadav SS, Dahiya K, Ganie SA et al., Antibacterial activity of Aegle marmelos (L) Correa, International Journal of Pharmacy and Pharmaceutical Sciences, 2015; 7(3):462-464.

25. Sircar B, Mandal S, Antibacterial activity of Mimusops elengi leaf seed and bark extracts Alone and in combination with antibiotics against human pathogenic bacteria, Translational Medicine (Sunnyvale), 2016; 6(4):188. DOI: 10.4172/21611025.1000188

26. Devi NP, Das SK, Sanjukta RK et al., A comparative study on antibacterial activity of integumentary extract of selected freshwater fish Species and Neem extracts against gram-positive and gram-negative bacteria, Journal of Entomology and Zoology Studies, 2019; 7(2):1352-1355.

27. Senapati S, Bagchi A, Raha A et al., Evaluation of antimicrobial activity of Azadirachta indica bark extract, The Pharma Innovation Journal, 2019; 8(6):691-694.

28. Singaravelu S, Sankarapillai J, Chandrakumari AS et al., Effect of Azadirachta indica crude bark extracts concentrations against gram-positive and gram-negative bacterial pathogens. Journal of Pharmacy and Bioallied Sciences, 2019; 11(1):33-37. DOI: 10.4103/jpbs.JPBS_150_18

29. Zihadi MAH, Rahman M, Talukder S et al., Antibacterial efficacy of ethanolic extract of Camellia sinensis and Azadirachta indica leaves on methicillin-resistant Staphylococcus aureus and shigatoxigenic Escherichia coli. Journal of Advanced Veterinary and Animal Research, 2019; 6(2):247-52. DOI: 10.5455/javar.2019.f340

30. Rezaie Keikhaie K, Fazeli-Nasab B, Jahantigh $\mathrm{HR}$ et al., Antibacterial activity of ethyl acetate and methanol extracts of Securigera Securidaca, Withania Sominefra, Rosmarinus Officinalis and Aloe vera plants against important human pathogens, Journal of Medical Bacteriology, 2018; 7 (1, 2):13-21.

31. Chepkorir R, Matasyoh JC, Wagara IN, Two withanolides from Withania somnifera (solanaceae) and activity of methanolic extracts against fungal and bacterial pathogens that affects food crops, African Journal of Food Science, 2018; 12(5):115- 125 DOI: $10.5897 /$ AJFS2016.1503

32. Verma M, Kumar A, Antimicrobial and antioxidant activity of whole plant extracts of Bacopa monnieri (1.) Pennell, International Journal of Applied Biology and Pharmaceutical Technology, 2017; 8(2):74-79. DOI: 10.21276/Ijabpt

33. Haque SKM, Chakraborty A, Dey $D$ et al., Improved micropropagation of Bacopa monnieri (L.) Wettst.
(Plantaginaceae) and antimicrobial activity of in vitro and ex vitro raised plants against multidrug-resistant clinical isolates of urinary tract infecting (UTI) and respiratory tract infecting (RTI) bacteria, Clinical Phytoscience 2017; 3(17):1-10. DOI 10.1186/s40816-017-0055-6

34. Mehmood S, Gull S, Mushtaq A et al., In vitro antibacterial and antioxidant activities of Santalum album and cymbopogon by sequential extraction, The Professional Medical Journal, 2019; 26(5):717-722. DOI: 10.29309/TPMJ/2019.26.05.3462

35. Singh HK, Charan AA, Charan AI et al., Antifungal and antibacterial activity of methanolic, ethanolic and acetonic leaf extracts of sarpagandha (Rauwolfia serpentine), Journal of Pharmacognosy and Phytochemistry, 2017; 6(5):152-156.

36. Kalita C, Saikia A, Sarma A et al., Antibacterial and antifungal property of three plants against oral microbes, Journal of Mahatma Gandhi Institute of Medical Sciences, 2018; 23:73-6. DOI: $10.4103 /$ jmgims.jmgims_4_16

37. Das MK, Mandal M, Mandal S, Assessment of bacterial growth inhibition property and phytochemical analysis of Ocimum sanctum L. leaf extract, International Research Journal of Pharmacy, 2017; 8(7):46-51. DOI: 10.7897/2230-8407.087116

38. Külcü DB, Gökışık CD, Aydın S, An Investigation of Antibacterial and Antioxidant Activity of Nettle (Urtica dioica L.), Mint (Mentha piperita), Thyme (Thyme serpyllum) and Chenopodium album L. Plants from Yaylacık Plateau, Giresun, Turkey, Turkish Journal of Agriculture - Food Science and Technology, 2019; 7(1):73-80. DOI: 10.24925/turjaf.v7i1.73-80.2123

39. Qidwai A, Pandey M, Shukla SK et al., Antibacterial activity of Mentha piperita and Citrus limetta against Propionibacterium acnes (anaerobic bacteria), International Journal of Pharmaceutical Sciences and Research, 2016; 7(7):2917-24. DOI: 10.13040/IJPSR.0975-8232.7 (7).2917- 24.

40. Zubair MF, Atolani O, Ibrahim SO et al., Chemical constituents and antimicrobial properties of Phyllanthus amarus (Schum \& Thonn), Bayero Journal of Pure and Applied Sciences, 2017; 10(1):238 - 246. DOI: $10.4314 /$ bajopas.v10i1.35

41. Sikdar S, Roy S, Banu TN, Phytochmical analysis and antibacterial property assessment of helencha (Enhydra fluctuans; Family: Asteraceae) extracts, GSC Biological and Pharmaceutical Sciences, 2020; 12(02):136-142. DOI: 10.30574/gscbps.2020.12.2.0252

42. Sircar B, Mandal S, Screening of Elaeocarpus floribundus fruit extracts for bioactive phytocomponents and antibacterial activity against food-borne bacteria. International Journal of Research in Medical Sciences, 2017; 5(8):3665-3671. DOI: 10.18203/2320-6012.ijrms20173582

43. Das MK, Mandal S, Syzygium cumini and Mangifera indica seed extracts: In Vitro assessment for antibacterial activity alone and in combination with antibiotics against clinical bacteria. Journal of Infectious Diseases \& Preventive Medicine, 2016; 4(1):129. DOI: $10.4172 / 2329-8731.1000129$

44. Banu TN, Mandal S, Antibacterial activity of Pomegranate (Punica granatum) fruit peel extracts against antibiotic resistant gramnegative pathogenic bacteria, Bioscience Biotechnology Research Communications, 2019; 12(4):1141-1149. DOI: $10.21786 / \mathrm{bbrc} / 12.4 / 38$

45. Kaho ZM, Kadum AR, Hadi AA. Evalution of antibacterial activity of Piper nigrum extract against Streptococcus mutans and Escherichia coli. Journal of Pharmaceutical Sciences and Research, 2019; 11(2):367-370.

46. Sreejai R, Raju A, Benchamin D et al., Comparative study of antimicrobial and phytochemical analysis of Piper longum and Piper nigrum, The Pharma Innovation Journal, 2019; 8(3):224-226.

47. Mandal M, Mandal S, Can Bacteriocins Curb the Emergence of Antibiotic Resistant Pathogenic Bacteria in the Globe?, Current Trends in Biomedical Engineering \& Biosciences, 2018; 17(3):555964. DOI: 10.19080/CTBEB.2018.17.555964.

48. Mahmoudi et al., Adhesion Properties of Probiotic Lactobacillus Strains Isolated from Tunisian Sheep and Goat Milk, Journal of Agriculture, Science and Technolog, 2019; 21(3):587-600

49. Kazemipoor et al., Screening of antibacterial activity of lactic acid bacteria isolated from fermented vegetables against food borne pathogens, Archives Des Sciences, 2012; 65(6):192-201.

50. Duangjitcharoen Y, Kantachote D, Ongsakul M, et al., Selection of Probiotic Lactic Acid Bacteria Isolated from Fermented Plant Beverages. Pakistan Journal of Biological Sciences, 2008;11:652655. DOI: $10.3923 /$ pjbs.2008.652.655 
51. Cotar et al., Quantitative real-time PCR study of the influence of probiotic culture soluble fraction on the expression of Pseudomonas aeruginosa quorum sensing genes, 2010; 69(4).

52. Chu et al., isolation and characterization of new potential probiotic bacteria based on quorum-sensing system, Journal of Applied Microbiology, DOI: 10.1111/j.1365-2672.2010.04872.x

53. Besser et al., Impact of probiotics on pathogen survival in an innovative human plasma biofilm model (hpBIOM), Journal of Translational Medicine, 2019; 7:243, DOI: 10.1186/s12967-0191990-4

54. Akbar A, Sadiq MB, Ali I, et al., Lactococcus lactis subsp. lactis isolated from fermented milk products and its antimicrobial potential, CyTA - Journal of Food, 2019; 17(1):214-220, DOI:10.1080/19476337.2019.1575474

55. Alang et al., Identification of lactic acid bacteria as antimicrobial from milk Toraja Belang buffalo, International Conference on Green Agro-industry and Bioeconomy, DOI:10.1088/1755$1315 / 230 / 1 / 012092$

56. Halder et al., Indigenous Probiotic Lactobacillus Isolates Presenting Antibiotic like Activity against Human Pathogenic Bacteria, Biomedicines, 2017; 5(31) DOI: 10.3390/biomedicines5020031

57. Halder D, Mandal S, Insights into the antagonism of Lactobacillus fermentum curd isolate against Gram-positive and Gramnegative pathogenic bacteria, Bioscience Biotechnology Research Communications, 2018; 11(3):461-468.

58. Patel A, Lindström C, Patel A, Prajapati J, Holst O, Probiotic properties of exopolysaccharide producing lactic acid bacteria isolated from vegetables and traditional Indian fermented foods. Internationa Journal of Fermented Foods, 2012; 1(1):87-101.

59. Cusimano MG, Spinello A, Barone G et al., A Synthetic derivative of antimicrobial peptide Holothuroidin 2 from Mediterranean Sea Cucumber (Holothuria tubulosa) in the control of Listeria monocytogenes, Marine Drugs, 2019; 17(159):1-11. DOI: $10.3390 / \mathrm{md} 17030159$

60. El-Sayed M, Awad S, Milk Bioactive Peptides: Antioxidant, Antimicrobial and Anti- Diabetic Activities, Advances in Biochemistry, 2019; 7(1):22-33. DOI: 10.11648/j.ab.20190701.15

61. Mandal SM, Silva ON, Franco OL, Recombinant probiotics with antimicrobial peptides: a dual strategy to improve immune response in immunocompromised patients, Drug Discovery Today, 2014; 19(8):1045-1050. DOI: 10.1016/j.drudis.2014.05.019

62. Ahmed TAE, Hammami R, Recent insights into structure-function relationships of antimicrobial peptides, Journal of Food Biochemistry, 2019; 43:e12546. DOI: 10.1111/jfbc.12546
63. Farhana et al., Isolation of Antimicrobial Peptide from Food Protein Hydrolysates: An Overview, Key Engineering Materials, 2019; 797:168-176.

64. Pottanat et al., Analysis of the Ribonuclease A Superfamily of Antimicrobial Peptides in Patients Undergoing Chronic Peritoneal Dialysis, Scientific reports, 2019; 9:7753 DOI: 10.1038/s41598-019-44219-x

65. Ferchichi M, Frere J, Mabrouk K et al. Lactococcin MMFII, a novel class IIa bacteriocin produced by Lactococcus lactis MMFII, isolated from a Tunisian dairy product. FEMS Microbiology Letters, 2001; 205:49-55.

66. Gong HS, Meng XC, Wang H, Mode of action of plantaricin MG, a bacteriocin active against Salmonella typhimurium, Journal of Basic Microbiology, 2010; 50(1):37-45. DOI 10.1002/jobm.201000130

67. Arumugam V, Venkatesan M, Ramachandran K et al., Purification, characterization and antibacterial properties of peptide from marine Ascidian Didemnum sp. International Journal of Peptide Research and Therapeutics, 2019; 26:201-208. DOI: 10.1007/s10989-019-09829-z

68. Freitas et al., Encrypted antimicrobial and antitumoral peptides recovered from a protein rich soybean (Glycine max) by-product, Journal of Functional Foods, 2019; 54:187-198.

69. Gao et al., Identification and antimicrobial activity evaluation of three peptides from laba garlic and the related mechanism, Food \& Function, DOI:10.1039/c9fo00236g

70. Li B, Lyu P, Xie S et al., LFB: A Novel Antimicrobial Brevinin-Like Peptide from the Skin Secretion of the Fujian Large Headed Frog, Limnonectes fujianensi, Biomolecules, 2019; 9(242):1-13. DOI: 10.3390/biom9060242

71. Fesenko I, Azarkina R, Kirov I et al., Phytohormone treatment induces generation of cryptic peptides with antimicrobial activity in the Moss Physcomitrella patens, BMC Plant Biology, 2019; 19(9):1-16. DOI: 10.1186/s12870-018-1611-z

72. Samriti, Biswas R, Biswas $K$, Antibacterial activity of antimicrobial peptide extracted from Trianthema portulacastrum Leaves, The Pharma Innovation Journal, 2019, 8(3):81-86.

73. Alexander $\mathrm{P}$, Oyama L, Huws $\mathrm{S}$, Utilising novel antimicrobial peptides isolated from the rumen microbiome as a treatment method for Acinetobacter baumannii, Queen's University Belfast. Access Microbiology, 2019; 1(1A):1. DOI: 10.1099/acmi.ac2019.po0043

74. Rončević T, Krce L, Gerdol $M$ et al., Membrane-active antimicrobial peptide identified in Rana arvalis by targeted DNA sequencing. Biochimica et Biophysica Acta (BBA) Biomembranes, 2018; 1861(3):651-659. DOI: 10.1016/j.bbamem.2018.12.014 